\title{
Surface Charge governs the adsorption behaviour of glyphosate in Australian Oxisol Soil System
}

Aman D. Sharma ( $\Delta$ aman.saluki@gmail.com )

Lovely Professional University https://orcid.org/0000-0001-5965-8868

\section{Research Article}

Keywords: Glyphosate Adsorption Pattern, Biochar, Batch Sorption Experiments, Potentiometric Titrations, Herbicide Soil Interactions

Posted Date: July 14th, 2021

DOl: https://doi.org/10.21203/rs.3.rs-702751/v1

License: (9) This work is licensed under a Creative Commons Attribution 4.0 International License. Read Full License 


\section{Abstract}

Glyphosate adsorption pattern is important for determining its environmental fate. Oxisol soil systems adsorb maximum glyphosate in the presence of biochar, however, the mechanism was not known. This article provides information concerning the role of surface charge in the case of Oxisol soil systems. Batch sorption experiments were conducted at the University of Sydney, Australia. Potentiometric titrations were done to calculate the surface charge in the case of Oxisol soil systems. Maximum adsorption of glyphosate in the Oxisol soil systems in the presence of char was due to an increase in net positive surface charge. This finding is of great importance to understanding the mechanism of herbicide soil interactions.

\section{Introduction}

Glyphosate (N-phosphonomethylglycine, $\mathrm{C}_{3} \mathrm{PNO}_{5} \mathrm{H}_{8}$ ) is a broad-spectrum herbicide. The molecule was introduced in 1974 by Monsanto and became the largest selling single crop protection chemical (Franz, Mao et al. 1997). Glyphosate has three functional groups (Amine, Carboxylate and Phosphonate) which are responsible for strong coordination with metal ions (Borggaard and Gimsing 2008). In soils, glyphosate sorption is by ligand exchange through the phosphonic acid moiety, a mechanism similar to phosphate adsorption (Gimsing and Borggaard 2007).

Glyphosate and phosphate ion compete for adsorption sites. Iron and aluminium oxides adsorb a greater amount of glyphosate and phosphates in comparison to clay silicates (Gimsing, Borggaard et al. 2004) and strong competition between glyphosate and phosphates for adsorption sites has been observed on surfaces of iron and aluminium oxides versus those of silicate clays indicating the role of soil mineralogy concerning glyphosate sorption. The adsorption process is important to assess the role of soil to act as a filter for protecting groundwater against pollution with glyphosate (Gimsing, Borggaard et al. 2004).

Bioavailability of herbicides can be greatly reduced with the application of biochar (Beesley, MorenoJimenez et al. 2010). The physical and chemical properties of biochar can affect the sorption behaviour of herbicides (Kookana, Sarmah et al. 2011). The adsorption behaviour of glyphosate has been reported in Australian soil systems (Gerritse, Beltran et al. 1996), however, for updated information on glyphosate behaviour, experiments were conducted at the University of Sydney, Australia. The adsorption behaviour of glyphosate in the presence and absence of biochar have been previously reported (Sharma and Lai 2019, Sharma 2020, Sharma, Singh et al. 2021), however, the present article would elucidate in detail the mechanism for the maximum adsorption of glyphosate in the Oxisol soil system in the presence of biochar.

\section{Materials And Methods}


This experiment was part of PhD study at the University of Sydney (Fang, Singh et al. 2014, Sharma and Lai 2019, Sharma 2020). As the Oxisol soil system showed maximum adsorption of glyphosate, potentiometric titration was used as a tool for surface charge determination (Lutzenkirchen, Preocanin et al. 2012) in this system. The potentiometric titration was done with 836 Titrando (Metrohm) involving strong acid (0.1 N Nitric Acid) in one dosing unit and $0.1 \mathrm{~N} \mathrm{NaOH}$ as a strong base in another dosing unit. Substrate involved $0.2 \mathrm{~g}$ (Soil / Soil+char) $+10 \mathrm{~mL}\left(1 \mathrm{M} \mathrm{KNO}_{3}\right)+90 \mathrm{~mL}$ (water). Calibration of the set up was done on a $\mathrm{pH}$ scale. $\mathrm{pH}$ was calibrated with known buffers and $0.025 \mathrm{~mL}$ of acid/base from the dosing units was added slowly in continuous iterations. To check for $\mathrm{CO}_{2}$ contamination, nitrogen gas was introduced. In continuous acid-base titration, dependency of the "equilibrium"-pH values of the colloid dispersion on the incremental added volume of titrant (strong acid/ strong base) was measured. The relative charge was obtained by plotting the $\mathrm{pH}$ as a function of the volume of the titrant.

\section{Statistical Analysis}

The batch experiments were repeated and the data were pooled as there was no significant interaction between treatments and experiments observed. The data were analysed using Analysis of variance (SAS 9.4) and the means were separated by Tukey's 95\% confidence intervals (Sharma and Lai 2019).

\section{Results And Discussion}

Titration curves Fig 1(a) and Fig 1(b) revealed that the surface charge in the Oxisol soil system was higher than the charge in the Oxisol soil system without char. This aspect explained the reason for the highest amount of glyphosate adsorbed in the Oxisol soil system (Fig. 1-2) (Sharma and Lai 2019, Sharma 2020). The presence of char increased the protonation and hence net surface charge became more positive on oxide surfaces; consequently, increasing the adsoption of anionic glyphosate (Sharma 2020). The finding is of great practical significance to understand soil-herbicide interactions.

\section{Conclusions}

Potentiometric titrations revealed that biochar increased the net positive surface charge in case of Oxisol soil systems and glyphosate being the anion adsorbed in higher amounts in these systems.

\section{Declarations}

The author duly acknowledges the financial support in the form of the University of Sydney International Scholarship (Student ID-440059389) and research support from the CSIRO. The authors declare that they have no conflict of interest.

\section{Data Availability Statement}

The datasets generated during and/or analysed during the current study are available from the corresponding author on reasonable request. 


\section{References}

Beesley, L., E. Moreno-Jimenez and J. L. Gomez-Eyles (2010). "Effects of biochar and greenwaste compost amendments on mobility, bioavailability and toxicity of inorganic and organic contaminants in a multi-element polluted soil." Environmental Pollution 158(6): 2282-2287.

Borggaard, O. K. and A. L. Gimsing (2008). "Fate of glyphosate in soil and the possibility of leaching to ground and surface waters: a review." Pest Management Science 64(4): 441-456.

Fang, Y., B. Singh, B. P. Singh and E. Krull (2014). "Biochar carbon stability in four contrasting soils." European Journal of Soil Science 65(1): 60-71.

Franz, J. E., M. K. Mao and J. A. Sikorski (1997). Glyphosate: a unique global herbicide.

Gerritse, R., J. Beltran and F. Hernandez (1996). "Adsorption of atrazine, simazine, and glyphosate in soils of the Gnangara Mound, Western Australia." Soil Research 34(4): 599-607.

Gimsing, A. L. and O. K. Borggaard (2007). "Phosphate and glyphosate adsorption by hematite and ferrihydrite and comparison with other variable-charge minerals." Clays and Clay Minerals 55(1): 108-114.

Gimsing, A. L., O. K. Borggaard and M. Bang (2004). "Influence of soil composition on adsorption of glyphosate and phosphate by contrasting Danish surface soils." European Journal of Soil Science $\mathbf{5 5 ( 1 ) :}$ 183-191.

Kookana, R. S., A. K. Sarmah, L. v. Zwieten, E. Krull and B. Singh (2011). "Biochar application to soil: agronomic and environmental benefits and unintended consequences." Advances in Agronomy 112: 103143.

Lutzenkirchen, J., T. Preocanin, D. Kovacevic, V. Tomisic, L. Lovgren and N. Kallay (2012). "Potentiometric Titrations as a Tool for Surface Charge Determination." Croatica Chemica Acta 85(4): 391-417.

Sharma, A. D. (2020). Crop competition as a strategy to control glyphosate-resistant Chloris virgata and application of biochar to control glyphosate mobility in Australian Soils Sydney eScholarship. https://hdl.handle.net/2123/21981

Sharma, A. D. and D. Lai (2019). "Sorption of radiolabelled glyphosate on biochar aged in contrasting soils." Journal of Environmental Science and Health, Part B 54(1): 49-53.

Sharma, A. D., B. Singh and R. Kookana (2021). "Use of Liquid Scintillation Counting to Study the Fate of C14 Labeled Glyphosate in Entisol Soil System." Communications in Soil Science and Plant Analysis: 1-7.

\section{Figures}



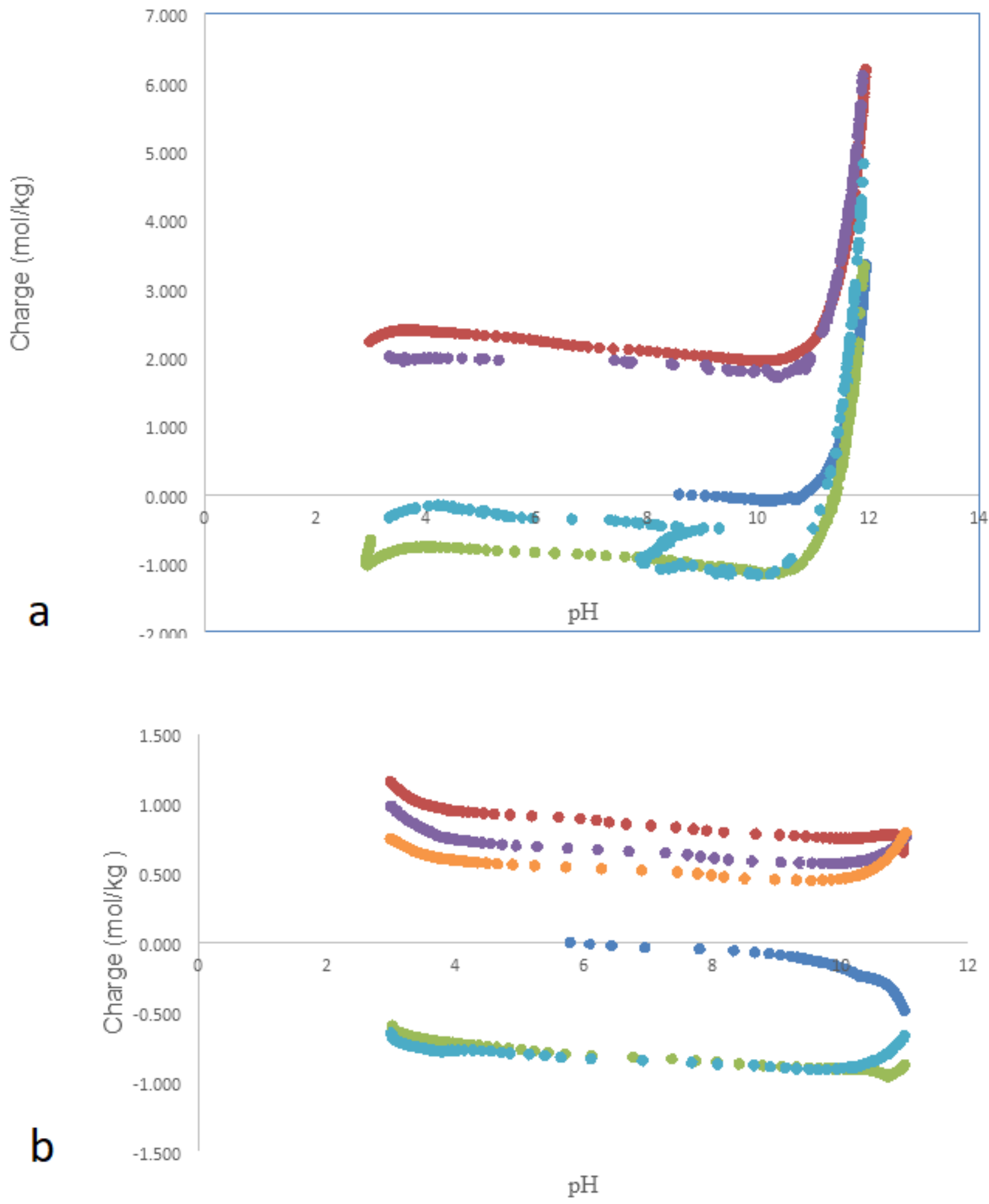

Figure 1

(a) Surface charge distribution in Oxisol soil system with char (b) Surface charge distribution in Oxisol soil system without char 